\title{
A REGURAL CURVE THAT CANNOT BE COVERED WITH FINITELY MANY CHORD-ARC CURVES
}

\author{
Lauri Kahanpää
}

\section{Introduction}

Generalizing a theorem by Hayman and Wu [HW], Fernandez and Hamilton in $[\mathrm{FH}]$ prove that every chord-arc curve in the plane has finite conformal length. Finite conformal length is a property inherited by subcurves and by finite unions of curves. In particular every finite union of chord-arc curves will have finite conformal length. A partial converse to this is the trivial observation that finite conformal length of a curve implies regularity in the sense of Alhfors [A1] and David [D2]. We give an example of a curve that is regular, yet not a subset of any finite union of chord-arc curves, not even of quasicircles.

Let $\gamma$ be a rectifiable, closed curve in the plane $\mathbf{R}^{2}=\mathbf{C}$. We will use the letter $\gamma$ to denote both an arc length parametrization $\gamma:[a, b] \rightarrow \mathbf{C}$, and the image set $\gamma=\gamma([a, b])$. The arc length distance between two points on $\gamma$ is understood as the shorter distance between them along $\gamma$, i.e. $l(\gamma(t), \gamma(s))=$ $\min \{|t-s|, b-a-|t-s|\}$. The corresponding arc length measure is also called $l$.

A curve $\gamma$ satisfies the chord-arc condition with constant $L$, if the arc length distance between points on $\gamma$ never exceeds $L$ times their ordinary distance:

$$
l\left(z_{1}, z_{2}\right) \leq L\left|z_{1}-z_{2}\right| \quad \text { for all } z_{1}, z_{2} \text { on } \gamma
$$

Chord-arc curves are also called Lavrentiev curves. A chord-arc curve is Jordan. Evidently it also satisfies the Ahlfors [A2] three points condition: "at least one of the arcs between any two points $z_{1}$ and $z_{2}$ on the curve has diameter at most a constant $C$ times $\left|z_{1}-z_{2}\right|$ ", characterizing quasicircles among (not necessarily rectifiable) Jordan loops. For details see [G].

A regular curve is a curve whose total length inside any circle $B\left(z_{0}, r\right)=\{z \in$ C ||$\left.z-z_{0} \mid \leq r\right\}$ never exceeds a constant $D$ times the radius $r$ of the circle:

$$
l\left(B\left(z_{0}, r\right) \cap \gamma\right) \leq D r \quad \text { for all } \quad z_{0} \in \mathbf{C}, r>0
$$

The chord-arc curves are precisely the regular quasicircles, but not all regular curves are such. A regular curve may well contain cusps or multiple points. The following example is intended to refute the conjecture that every regular curve is 


\section{Lauri Kahanpää}
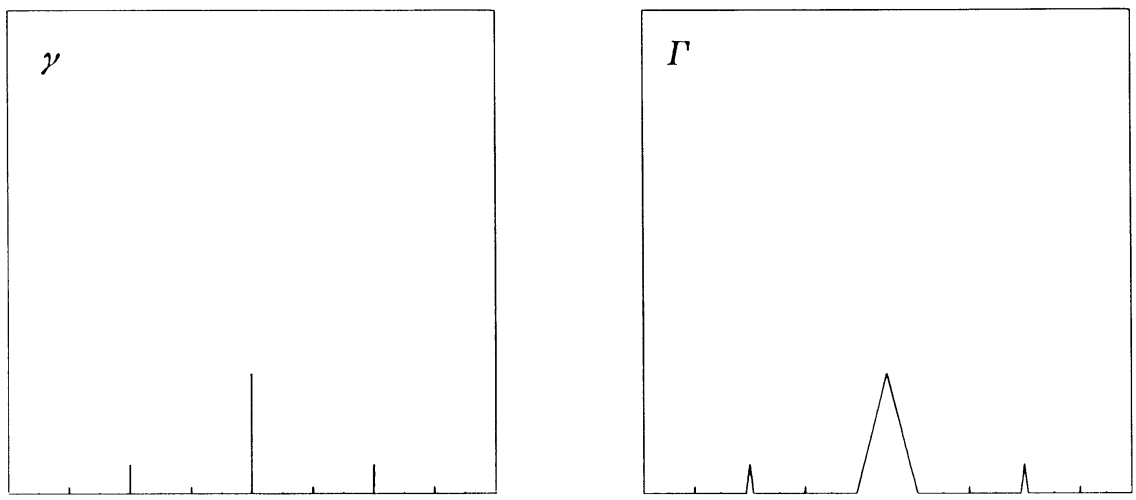

Figure 1.

at least contained in the union of finitely many quasicircles. Two variants of the example will be presented, the first being easier to handle, the second having the additional property of being a Jordan loop.

Instead of writing down a formal definition of the curves in question we prefer to describe them using Figure 1, and a few explanatory words.

\section{The simpler example}

The set $\gamma$ consists of the boundary of the unit square and of vertical lines, one for each dyadic rational point on the base interval of our square. These should become shorter with increasing order of the corresponding dyadic rational. We choose height $2^{-2 m}$ at $p / 2 m, p$ odd, as indicated in Figure 1 . There is a natural parametrization of $\gamma$ as a closed rectifiable curve passing twice through every point on these lines and once through the remaining points on the boundary of the unit square. The interesting part of $\gamma$ is the arc consisting of the base interval of the square and of the vertical lines, call it the basic arc. Any subarc of it between two distinct real points $(x, 0)$, and $\left(x^{\prime}, 0\right)$ contains a miniaturized and dilated copy of the basic arc. This is now used to demonstrate that $\gamma$, obviously regular, cannot be covered using finitely many chord-arc curves. Assume the contrary. Without loss of generality we may think of $\gamma$ as being covered with as few as possible such curves $\gamma_{1}, \gamma_{2}, \ldots, \gamma_{n}$. As the same is true with respect to the miniaturized and dilated copies of the basic arc no subarc of $\gamma$ between two distinct real points can be covered using less than $n$ of the curves $\gamma_{1}, \gamma_{2}, \ldots, \gamma_{n}$, i.e. all of them. In particular every $\gamma_{k}$ is dense on the unit interval of the real line and so by compactness each $\gamma_{k}$ contains the unit interval. On the other hand the union of the curves $\gamma_{k}$ does also cover the vertical lines used in the construction of $\gamma$. Therefore, by finiteness at least one of the curves $\gamma_{k}$ contains a sequence of points outside the real line converging to $(1 / 2,0)$, say. But it is easy to see and will follow from the lemma in Section 3 that no quasicircle can contain both an interval and 
a sequence of points outside that interval converging to any internal point of the interval.

\section{The Jordan loop example}

We start the construction with a Cantor set

$$
F=[0,1] \backslash \bigcup_{m=0}^{\infty} \bigcup_{k=1}^{2^{m}} E_{m, k}
$$

where the open intervals $E_{m, k}$ deleted at the $m$ th stage have length $l_{m}=l\left(E_{m, k}\right)$ $=2^{-3 m-3}$. The curve $\Gamma$ will now consist of the boundary of the unit square with the real unit interval replaced by the graph of the continuous function

$$
f:[0,1] \rightarrow[0,1 / 4]: x \rightarrow \begin{cases}2^{-2 m} \operatorname{dist}(x, F) / \frac{1}{2} l_{m}, & \text { if } x \in E_{m, k}, \\ 0, & \text { if } x \in F .\end{cases}
$$

Obviously $\Gamma$ is regular. To prove that $\Gamma$ cannot be covered with finitely many quasicircles we have to modify the proof given for the first version of the example, since now $\Gamma$ does not contain any subinterval of the real line the real points of $\Gamma$ forming a Cantor set.

Lemma. If $\sigma$ is a quasicircle in the plane $\mathbf{C}$ passing through an imaginary point $b i, b>0$, and through some real points $a_{1}<a_{1}<\cdots<a_{n}$, then the three points constant $C$ of $\sigma$ is at least equal to $\min \left\{b / d,-a_{0} / 2 b, a_{n} / 2 b\right\}$, where $d$ is the norm of the partition $\left.a_{0}, \ldots, a_{n}\right)$, i.e. $d=\max \left\{a_{j}-a_{j-1} \mid j=1, \ldots, n\right\}$.

Proof. By the three points property for every $z$ on one of the two arcs between $a_{j-1}$ and $a_{j}$ :

$$
|\operatorname{Im} z| \leq C\left|a_{j-1}-a_{j}\right| \leq C d .
$$

Hence either $b \leq C d$-in which case we are done-or $\sigma$ cannot pass through $b i$, not even leave the strip $|\operatorname{Im} z| \leq b$ on one of its arcs between any points $a_{j-1}$ and $a_{j}, j=1, \ldots, n$. The union of such arcs is an arc of $\sigma$ joining $a_{0}$ and $a_{n}$ without ever leaving the strip $|\operatorname{Im} z| \leq C d$. We assume $a_{0}<0<a_{n}$, since the alternative case is trivial. Thus our arc passes through some imaginary point $c i$ with $|c| \leq C d \leq b$. On the other hand the complementary arc of $\sigma$ passes through $b i$. Now one of the arcs between $b i$ and $c i$ contains $a_{0}$ and the other $a_{n}$, so they have diameters at least $-a_{0}$ and $a_{n}$, respectively. But $|b i-c i| \leq b+|c| \leq 2 b$, so

$$
\min \left\{-a_{0}, a_{n}\right\} \leq 2 C b,
$$

which completes the proof of the lemma.

Let us now continue our examination of the Jordan loop $\Gamma$. Assume that $\Gamma$ is covered with minimally many chord-arc curves $\gamma_{1}, \gamma_{2}, \ldots, \gamma_{n}$. By minimality 
of $n$ and compactness of $\gamma_{k}$ every $\gamma_{k}$ contains the Cantor set $F$, in particular every $\gamma_{k}$ contains the endpoints of all the deleted intervals $E_{m, j}$. Choose some $E_{m, j}$, call its midpoint $x_{m, j}$ and assume $m$ is large enough, $m \geq 10$ say. The distance $\operatorname{dist}\left(x_{m, j}, E_{m^{\prime}, j^{\prime}}\right)$ for $m^{\prime} \leq m,(m, j) \neq\left(m^{\prime}, j^{\prime}\right)$, will then be larger than $2^{-m} / 5$. Thus the set $\left\{x \in[0,1] \mid x \notin F, \operatorname{dist}\left(x, x_{m, j} \leq 2^{-m} / 10\right\}\right.$ does not contain any interval of length more than $l_{m}<2^{-3 m}$. At least one $\gamma_{k}$ passes through the point $\left(x_{m, j}, 2^{-2 m}\right)$. Translating $x_{m, j}$ to the origin we may apply the lemma to $\gamma_{k}$ choosing $b=2^{-2 m}, d \leq 2^{-3 m}$ and both $-a_{0}$ and $a_{m} \geq 2^{-m} / 20$. We find $C \geq 2^{m} / 40$ which may be made arbitrarily large in contradiction to the finite number of possible choices of $\gamma_{k}$. .

We finally point out that replacing successively all intervals on $\gamma$ with copies of the basic arc will in the limit lead to a curve, no proper subarc of which can be embedded in the union of finitely many quasicircles.

\section{Another property of the curves $\gamma$ and $\Gamma$}

In a preprint to appear ([L]) V. Lappalainen proves that the bounded domain $D$ with boundary $\gamma$ (or $\Gamma$ ) is a John domain which cannot be expressed as the union of finitely many quasiconvex domains. (In fact, it was through this work of Lappalainen's that the author's interest was directed to these curves.)

\section{References}

[A1] Ahlfors, L.V.: Zur Theorie der Überlagerungsflächen. - Acta Math. 65, 1935, 157-194.

[A2] AhLFors, L.V.: Quasiconformal reflections. - Acta Math. 109, 1963, 291-301.

[D1] David, G.: Courbes corde-arc et espaces de Hardy généralisés. - Ann. Inst. Fourier (Grenoble) $32,1982,227-239$.

[D2] DAVID, G.: Opérateurs intégraux singuliers sur certaines courbes du plan complexe. - Ann. Sci. École Norm. Sup. $4^{\text {e }}$ série 17, 1984, 157-189.

[FH] Fernandez, J.L., and D.H. Hamilton: Lengths of curves under conformal mappings. Comment. Math. Helv. 62, 1987, 122-134.

[G] Gehring, F.W.: Characteristic properties of quasidisks. - Les Presses de l'Université de Montréal, 1982.

[HW] Hayman, W.K., and G. WU: Level sets of univalent functions. - Comment. Math. Helv. $56,1981,366-403$.

[L] Lappalainen, V.: A John domain that is not the union of finitely many uniform domains. - Preprint, University of Jyväskylä, Department of Mathematics (to appear).

University of Jyväskylä

Department of Mathematics

Seminaarinkatu 15

SF-40100 Jyväskylä

Finland

Received 28 April 1988 